\title{
Clinical outcomes and lipid-laden macrophages in Electronic cigarette/Vaping product use-Associated Lung Injury (EVALI) in a South Texas population
}

\author{
Mahnoor Mir ${ }^{1}$, Moeezullah Beg ${ }^{2}$, Jay I. Peters ${ }^{2,3}$, Sandra G. Adams*2,3 \\ ${ }^{1}$ Department of Medicine, UT Health San Antonio, San Antonio, TX, USA \\ ${ }^{2}$ Department of Medicine, Pulmonary/Critical Care Division, UT Health San Antonio, San Antonio, TX, USA \\ ${ }^{3}$ South Texas Veterans Health Care System, San Antonio, TX, USA
}

Received: January 3, 2021

Accepted: April 29, 2021

Online Published: May 24, 2021

DOI: $10.5430 /$ dcc.v8n1p1

URL: https://doi.org/10.5430/dcc.v8n1p1

\begin{abstract}
Objective: To describe the clinical features, proportion of lipid-laden alveolar macrophages in bronchoalveolar lavage (BAL), and short-term and 6-month to 12-month outcomes of patients with Electronic cigarette/Vaping product use-Associated Lung Injury (EVALI).

Methods: Retrospective review of clinical characteristics, radiographs, and BAL samples for all patients with a history of vaping who presented with acute hypoxemic respiratory failure to the University Hospital in San Antonio, Texas from 9/2019 to 6/2020 was performed.

Results: We report 16 cases (15 men; median age, 30 years [range 19-75]) of EVALI with a history of vaping Tetrahydrocannabinol (THC), nicotine, or both. The most common presenting symptoms were tachycardia, dyspnea, cough, and fever. All patients required supplemental oxygen, including two who required noninvasive positive pressure ventilation, and five who required mechanical ventilation. All 16 patients had bilateral ground-glass opacities (GGO) with peripheral sparing on chest computerized tomography (CT). Cultures were negative, except for one patient who tested positive for rhinovirus. COVID-19 PCR was done in one individual which was negative. Cytology demonstrated lipid-laden macrophages on Oil-Red-O stain on fresh (i.e., without fixative) BAL in the majority of patients $(\mathrm{N}=12)$ with a mean lipid-saturation percentage of $78 \%$ [range, $44 \%-100 \%$ ] and the mean Colombo count of 194 [range, 101-359]. Fifteen patients were treated with systemic corticosteroids. The median length of hospital stay was 10 days. At discharge, three patients required supplemental oxygen. Eight of those who had follow-up imaging showed resolution of GGO. One patient had a relapse of symptoms and was again treated with systemic corticosteroids and mycophenolate, with resolution of symptoms. Fourteen patients who were evaluated after discharge denied vaping post-discharge (two patients were not able to be contacted and did not keep follow-up appointments).

Conclusions: Successful diagnosis and management of EVALI requires a high clinical suspicion, thorough evaluation to rule out infectious etiologies, and aggressive treatment with systemic corticosteroids, along with sustained abstinence from vaping.
\end{abstract}

Key Words: EVALI, Vaping, Acute lung injury, Lipid-laden macrophages, Diffuse ground glass opacities

*Correspondence: Sandra G. Adams; Email: adamssg@uthscsa.edu; Address: 7400 Merton Minter (111E), San Antonio, TX 78229, USA. 


\section{INTRODUCTION}

Electronic cigarettes are battery-operated devices that have a cartridge containing liquid, (nicotine, THC, or cannabis) and an atomizer (vaporization chamber with a heating element) (see Figure 1). ${ }^{[1]}$ Electronic-cigarette or vaping, product useassociated lung injury (EVALI) was first reported to the Centers for Disease Control and Prevention in the United States in August 2019 and has led to a significant number of hospitalizations and deaths. The CDC reported a total of 2,807 hospitalized EVALI cases and 68 deaths, with a majority of cases being associated with tetrahydrocannabinol-containing products. ${ }^{[2]}$ Texas has the highest reported EVALI cases or deaths per state in the country, almost 250 cases have been reported by February 2020. ${ }^{[2]}$

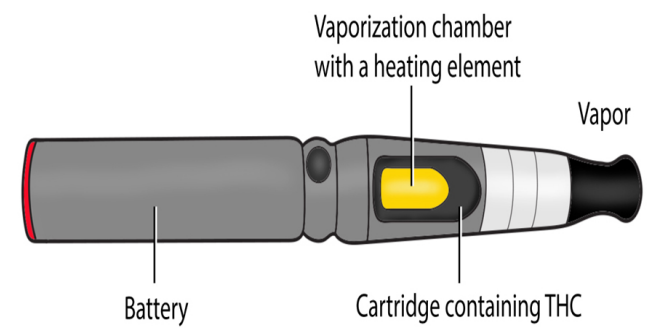

Figure 1. Components of an electronic cigarette

Various patterns of acute lung injury including diffuse alveolar damage, acute fibrinous pneumonitis, and organizing pneumonia have been described in association with EVALI. ${ }^{[3,4]}$ In addition, the lipid-laden macrophages (LLM) in bronchoalveolar cell lavage (BAL) has been noted in some series of EVALI. ${ }^{[3,4]}$ Although EVALI cases continue to be reported, it remains a diagnosis of exclusion and is without a specific confirmatory diagnostic test. EVALI and COVID-19 can have an overlap of clinical symptoms, like respiratory and constitutional symptoms along with ground glass opacities on imaging, however, our study includes only one patient who was tested negative for COVID-19 in May 2020, due to the remaining patients presenting prior to the first known COVID-19 positive patient in our area. ${ }^{[5]}$

We present a case series to describe the outcomes in the acute phase of the illness as well as the recovery and residual effects present 6-12 months following the initial hospitalization. We will also discuss the presenting signs and symptoms, hospital course, the prevalence and significance of LLM in bronchoalveolar lavage, the management of EVALI, and our strategy for follow-up after discharge.

\section{MethodS}

We collected and reviewed patients with a personal history of vaping or exposure to vaping who presented to the University
Hospital or the South Texas Veterans Health Care System (VA Hospital) in San Antonio, Texas from 9/2019 to 6/2020 with acute symptoms and who were ultimately diagnosed with EVALI. We report the clinical characteristics, radiographs, BAL samples, clinical characteristics at discharge and as well as symptoms, pulmonary function testing and radiographic imaging 6-12 months after discharge.

\section{RESULtS}

Of the 16 cases of EVALI ( 15 men; median age, 30 years, range 19-75) seen at University Hospital and the South Texas Veterans Health Care System in San Antonio, Texas between 9/2019 to 6/2020, 15 reported a history of vaping Tetrahydrocannabinol (THC) with or without nicotine and one reported recurrent passive exposure as a transportation driver who regularly allowed vaping in his vehicle. Ten patients had no significant medical history, five had a history of asthma and/or COPD, and one had undergone bilateral lung transplantation 19 years before presentation with EVALI. All but one patient had respiratory symptoms at presentation.

\subsection{Vital signs, symptomatology}

The most common presenting symptoms were tachycardia $(\mathrm{N}=13,81 \%)$, dyspnea $(\mathrm{N}=12,75 \%)$, cough $(\mathrm{N}=12,75 \%)$, productive cough $(\mathrm{N}=5,33 \%)$, chest pain $(\mathrm{N}=6,38 \%)$, gastrointestinal symptoms (including nausea, vomiting, and/or diarrhea) $(\mathrm{N}=8,50 \%)$, and fever $(\mathrm{N}=10,67 \%)$ and occurred for a median of 7 days before admission (see Table 1). On admission, six (38\%) were febrile and $13(81 \%)$ presented with acute hypoxemic respiratory failure and all patients progressed to acute hypoxemic respiratory failure during the hospital stay requiring supplemental oxygen, including seven who required high-flow nasal cannula, two who required noninvasive positive pressure ventilation (NPPV), and five who required invasive mechanical ventilation (see Table 1).

\subsection{Radiographic findings}

Initial imaging demonstrated diffuse bilateral opacities on chest radiograph (see Figure 2) and all patients had diffuse bilateral ground-glass opacities sparing the periphery of the lungs on chest computerized tomography (CT) imaging (see Figure 3).

\subsection{Investigations, clinical course \& treatment}

All patients endorsed THC vaping history except the one mentioned above who reported repeated exposure to passengers who were vaping in his vehicle during transport to their destination. The history of vaping varied from 2 months up to 5 years, with some patients endorsing use of dabbing with butane and "dank" vapes from California. Only six patients 
$(38 \%)$ endorsed smoking marijuana and four $(25 \%)$ endorsed the use of traditional tobacco cigarettes (see Table 1).

Due to high clinical suspicion of EVALI, bronchoscopy was performed in all patients within 3-4 days (median 3 days) of admission and cytology on fresh, non-fixed BAL fluid demonstrated LLM on the Oil-Red-O stain in 12 patients (not present in two patients and not evaluated in two patients), with a mean lipid saturation percentage of $78 \%$ [range, $44 \%$ 100\%] and the mean Colombo count was 194 [range 101359] (see Figure 4). One procedure was aborted due to acute severe hypoxemia. The other patient requested and arranged transfer to a different hospital, where an Oil-Red-O stain was not performed, but transbronchial biopsies demonstrated organizing pneumonia (see Table 2). Sputum, BAL, and blood cultures were negative in all patients, except for one with rhinovirus/enterovirus. All patients were treated with variable doses of systemic corticosteroids and tapered as an outpatient. Time to initiation of steroids was also variable, 11 patients $(73 \%)$ received steroids before, or the same day of the bronchoscopy, and 4 patients $(26.6 \%)$ received corticosteroids after the bronchoscopy specimen stains were negative for active infection. The difference in timing of initiation of corticosteroids was to rule out other underlying infectious causes.

Table 1. Patients' characteristics

\begin{tabular}{|c|c|}
\hline Items & Total $N=16(\%)$ \\
\hline Median age (years) & 30 \\
\hline \multicolumn{2}{|l|}{ Sex } \\
\hline Male & 15 \\
\hline Female & 1 \\
\hline Initial respiratory symptoms & $15(94)$ \\
\hline Cough & $12(75)$ \\
\hline Productive cough & $5(31)$ \\
\hline Shortness of breath & $12(75)$ \\
\hline Chest pain & $6(38)$ \\
\hline Fever & $10(63)$ \\
\hline Gastrointestinal symptoms (Nausea, vomiting, and/or diarrhea) & $8(50)$ \\
\hline Past Medical History (DM2, CAD, Hypothyroidism) & $7(44)$ \\
\hline History of pulmonary disease (COPD, Asthma, Lung transplant) & $4(25)$ \\
\hline Median Symptoms duration before hospital presentation & 7 days \\
\hline \multicolumn{2}{|l|}{ Substance abuse History } \\
\hline Tetrahydrocannabinol vaping & $16(100)$ \\
\hline Marijuana smoke & $6(38)$ \\
\hline Tobacco cigarettes & $4(25)$ \\
\hline \multicolumn{2}{|l|}{ Initial Vitals on Presentation } \\
\hline Heart rate $>100$ beats per minute & $13(81)$ \\
\hline Temperature $>100.4$ degrees Fahrenheit & $6(38)$ \\
\hline Systolic blood pressure $<90 \mathrm{mmHg}$ & 0 \\
\hline Oxygen Saturation $<92 \%$ on room air & $13(81)$ \\
\hline \multicolumn{2}{|l|}{ Respiratory interventions } \\
\hline Supplemental Oxygen (at least low-flow nasal cannula) & $16(100)$ \\
\hline High-flow nasal cannula & $7(44)$ \\
\hline Noninvasive positive pressure ventilation & $2(13)$ \\
\hline Mechanical Ventilation & $5(31)$ \\
\hline Intensive care admission or transfer during hospital stay & $6(38)$ \\
\hline Bronchoscopy performed & $16(100)$ \\
\hline Bronchoalveolar lavage with Oil-Red-O stain performed & $87.5 \%$ \\
\hline Median length of hospital stay & 10 \\
\hline
\end{tabular}




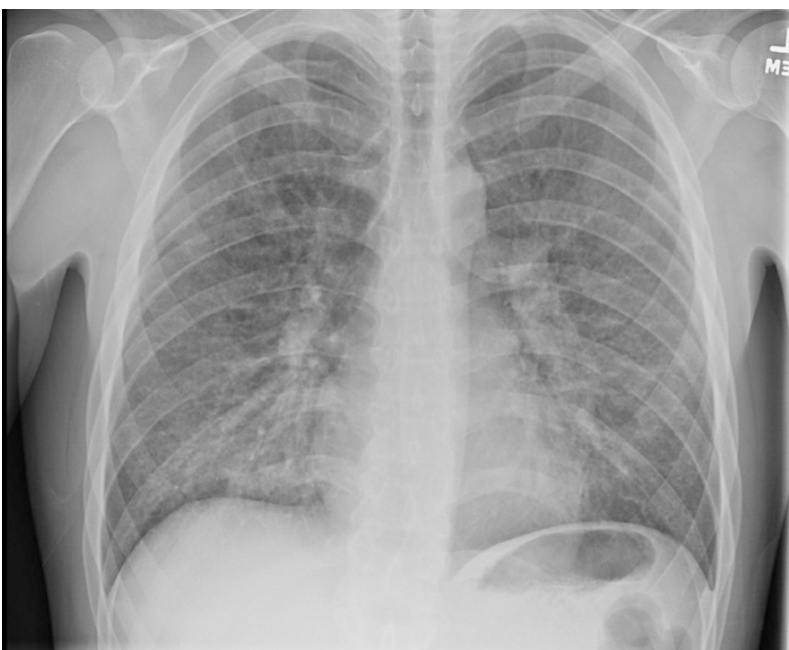

Figure 2. Chest radiograph showing diffuse bilateral pulmonary opacities

The median length of hospital stay was 10 days [range 4-25 days]. Three of these patients (19\%) continued to require supplemental oxygen therapy after discharge. Before discharge, all patients were counseled about the importance of avoiding inhaling any substances into the lungs via e-cigarettes, THC, tobacco via traditional cigarettes, etc.

Table 2. Bronchoscopy findings

\begin{tabular}{ll}
\hline Procedure & Total N (\%) \\
\hline Bronchoscopy performed & $16 / 16(100)$ \\
Lipid-Laden Macrophages $^{*}$ & $12 / 14(86)$ \\
Mean Lipid saturation percentage & $78 \%$ [range 44\%-100\%] \\
Mean Colombo count on Oil-Red-O Stain & 183 [range 101-359] \\
$\begin{array}{l}\text { Other findings: } \\
\text { Organizing pneumonia }\end{array}$ & $1(6)$ \\
Rhinovirus/Enterovirus & $1(6)$ \\
\hline $\begin{array}{l}\text { Note. }{ }^{*} \text { Oil-Red-O staining not performed in 2 cases, one aborted due to hypoxemia and } \\
\text { one underwent bronchoscopy at another facility }\end{array}$
\end{tabular}
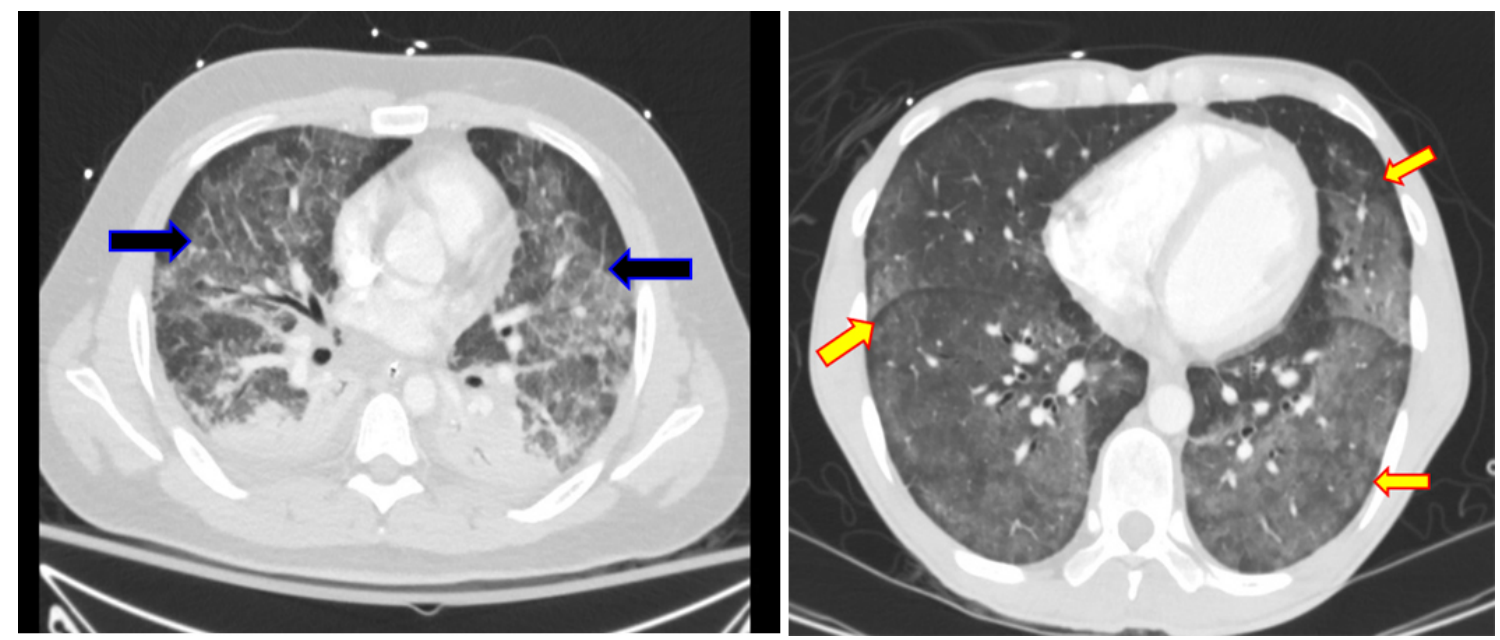

Figure 3. Computed tomography of the chest showing inter-lobular septal thickening (black arrows) along with multi-focal bilateral hazy ground-glass opacities sparing the periphery (yellow arrows)

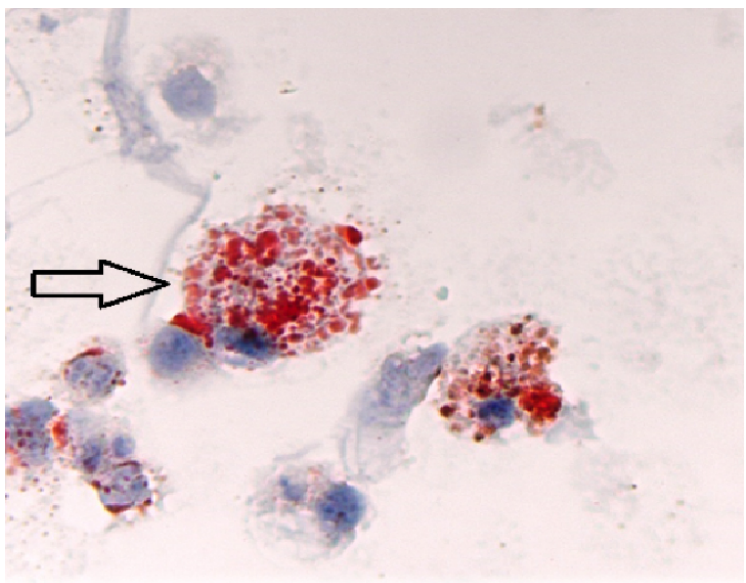

Figure 4. Bronchoalveolar lavage fluid with Oil-Red-O stain demonstrating multiple lipid-laden macrophages

\subsection{Interval follow up}

In addition to counseling patients about the importance of vaping abstinence, the need for follow up chest imaging and pulmonary function tests in 2-6 months was stressed. Two patients did not keep follow up appointments and had disconnected phone numbers; therefore, were not able to be contacted. Fourteen of the 16 patients with EVALI reported sustained abstinence from electronic cigarettes throughout their follow up period. Twelve patients reported complete symptom resolution following the corticosteroid taper at home. One patient was discharged on HFNC, oral corticosteroids and mycophenolate had a relapse of symptoms after about 6 months despite no additional exposure to electronic cigarettes. This patient was again treated with pulse-dose systemic corticosteroids and oral mycophenolate. After slowly 
tapering therapy, all symptoms resolved over a four-month period of observation. One additional patient had residual ground glass opacities on chest CT at a two-month follow-up, which resolved completely at the six-month follow-up visit. He continued to report bothersome dyspnea with significant exertion, which completely resolved spontaneously approximately 10 months after discharge. Two of the five patients who underwent PFTs post-discharge demonstrated mild restrictive physiology, one patient had a moderate reduction in diffusion capacity, and the remaining two patients had normal PFTs. Limitations to follow up in the clinic and poor compliance with imaging and PFT follow up were likely due to young age and lack of significant medical history.

\section{Discussion}

In the last decade, electronic cigarettes, vaping initially emerged as an alternative to conventional cigarettes \& nicotine use, gaining popularity amongst healthy young adults. It emerged as a health hazard in 2019 due to its potentially harmful effects observed in the consumers. A total of 2,807 hospitalized EVALI cases with 68 reported deaths have been confirmed by the Centers for Disease Control and Prevention in the United States with most cases being associated with tetrahydrocannabinol containing products. Texas has the highest reported EVALI cases or deaths per state in the country, 200-249 cases until February 2020.2 Most patients with EVALI report the use of products containing Tetrahydrocannabinol (75 to 80 percent). ${ }^{[6]}$

Cannabis (also called marijuana) is the illegal substance in many states that is most commonly used worldwide ${ }^{[7]}$ through pulmonary (smoked or inhaled vapor) or oral routes. Nicotine and tetrahydrocannabinol (THC, 5\%-15\% active ingredient in marijuana) are the two main substances vaped followed by the use of Cannabidiol (CBD) and other cannabinoids for vaping. "Dabbing" is a related activity where a user will perform an extraction of marijuana typically with butane or another organic solvent to produce a highly concentrated extract that can be inhaled, smoked, or used in an e-cigarette device. ${ }^{[8]}$

EVALI should be suspected in patients with a history of vaping or other electronic cigarette-related products with respiratory symptoms (cough, dyspnea, fever) with acute hypoxemia and no infectious etiology. The histopathological features involve multiple spectrums of disease processes including acute fibrinous pneumonitis, diffuse alveolar damage, lipoid pneumonia, and organizing pneumonia. ${ }^{[3,4]}$ Radiographic abnormalities including interlobular septal thickening along with bilateral hazy ground-glass opacities have also been described with this condition. LLM in bronchoalveolar lavage fluid has been noted in many patients with EVALI, but Published by Sciedu Press the significance of LLM in diagnosing this condition remains unclear. ${ }^{[9,10]}$ LLM presence in EVALI was first noted in a case of acute lung injury in 2012, and over $80 \%$ of BAL samples evaluated with Oil-Red-O stain identified LLM on bronchoscopy for the cases published in 2019. ${ }^{[8]}$

However, the presence of LLM in a patient with a history of electronic-cigarette or vaping product use and no evidence of infection should strongly suggest a diagnosis of EVALI. Our case series reports a higher percentage of LLM than previously reported. ${ }^{[3]}$ EVALI is still a diagnosis of exclusion and can sometimes be a late diagnosis or missed diagnosis; hence timely administration of systemic corticosteroids and confirmation with bronchoscopy has shown better outcomes with resolved acute hypoxemia and complete resolution of symptoms in 3-6 months.

There is a certain limitation in using LLM as a diagnostic tool, findings of foamy LLM in BAL fluid are not specific for aspirated or inhaled lipid ${ }^{[11]}$ and can be seen in many cases of acute lung injury, regardless of the cause. Shields et al, showed high LLM in the lungs of all conventional smokers and e-cigarettes users, however LLM presence in vape pen users was further associated with elevated levels of the inflammatory cytokines IL-4 and IL-10 in bronchoalveolar lavage fluid. ${ }^{[12]}$

Vitamin E acetate is a thickening chemical agent which is used in THC containing products. Blount et al. ${ }^{[13]}$ showed Vitamin E in $94 \%$ of patients with THC associated EVALI, an experimental study ${ }^{[14]}$ in rodents has shown EVALI symptoms without the use of THC, vitamin $\mathrm{E}$ or nicotine use. However, all of our patients used THC products that were obtained from out of state with little to no knowledge about THC packing/preparation. Further investigational studies in humans need to be done to find an association of EVALI without the use of THC, nicotine, or vitamin $\mathrm{E}$ in vaping products.

There is a paucity of data reporting the long-term patient outcomes after admission for EVALI, particularly related to the long-term effects on respiratory symptoms, pulmonary function tests, imaging, and relapse. Some patients with EVALI may have long-term restrictive physiology on PFTs and recurrence of symptoms even when vaping is discontinued.

\section{Conclusion}

THC is one of the common vaping agents used in the United States. We present a case series describing our diagnosis, management and 6-12 months follow up of patients with EVALI based on maintaining a high clinical suspicion, performing appropriate diagnostic testing including labs, imag- 
ing, and bronchoscopy with BAL stains for LLM as well as the usual studies to rule out infections, and then treating early and aggressively with systemic corticosteroids. LLM on the Oil-Red-O stain with a high Colombo count ${ }^{[15]}$ was found in the bronchoscopy of the majority of patients with a history of vaping without any other underlying cause of acute hypoxemic respiratory failure.

To date, no laboratory tests have been identified to confirm EVALI. Maintaining a high clinical suspicion and performing BAL with lipid stains on specimens without fixative in the appropriate clinical setting may assist clinicians in early diagnosis and aggressively managing these patients. EVALI and COVID-19 share clinical and radiographic findings, however, due to limitations to aerosolizing procedures with COVID-19 use of bronchoscopy with BAL will be a leading clue for the diagnosis of EVALI like LLM and vitamin E on BAL. To date, less is known about COVID BAL findings. The loss of taste and smell described in patients with COVID-19 was not present and has not been described in the literature or in any of our patients with EVALI.

Limitations to follow up imaging in the clinic may be attributed to financial limitations as well as young age and no prior medical problems. Counseling about vaping cessation is also critical for these patients.

\section{Clinical implications}

- Currently, no test has been identified to confirm EVALI. Diagnosis is based on a high clinical suspicion in patients with vaping exposure, as well as obtaining classic imaging and laboratory tests to rule out other potential etiologies.

- Bronchoscopy and BAL fluid without fixative stained with Oil-Red-O in the appropriate clinical setting may assist clinicians in early diagnosis and in decisions regarding initiating aggressive corticosteroid therapy in these patients.

- Interval follow-up with chest imaging, PFTs, and ongoing counseling about sustained abstinence for vaping is also critical for these patients.

- Further studies need to be done to establish clinical diagnostic differences between EVALI and COVID-19.

\section{CONFlicts OF InTEREST Disclosure}

The authors declare they have no conflicts of interest.

\section{REFERENCES}

[1] Hartmann-Boyce J, Begh R, Aveyard P. Electronic cigarettes for smoking cessation. BMJ. 2018; 360: j5543. PMid:29343486. https : //doi.org/10.1136/bmj.j5543

[2] Centers for Disease Control and Prevention. Outbreak of Lung Injury Associated with the Use of E-Cigarette, or Vaping, Products 2020. Available from: www.cdc.gov/tobacco/basic_information/ e-cigarettes/severe-lung-disease.html

[3] Guerrini V, Panettieri RA, Jr., Gennaro ML. Lipid-laden macrophages as biomarkers of vaping-associated lung injury. Lancet Respir Med. 2020; 8(2): e6. PMid:32035068. https ://doi .org/10.1016/S2 213-2600 (19) 30476-X

[4] Kalininskiy A, Bach CT, Nacca NE, et al. E-cigarette, or vaping, product use associated lung injury (EVALI): case series and diagnostic approach. Lancet Respir Med. 2019; 7(12): 1017-26. PMid:31711871. https ://doi .org/10.1016/S2213-2600(19) 30415-1

[5] Callahan SJ, Harris D, Collingridge DS, et al. Diagnosing EVALI in the Time of COVID-19. Chest. 2020; 158(5): 2034-7. PMid:32599069 https://doi.org/10.1016/j. chest.2020.0 6.029

[6] Heinzerling A, Armatas C, Karmarkar E, et al. Severe Lung Injury Associated With Use of e-Cigarette, or Vaping, Products-California, 2019. JAMA Intern Med. 2020; 180(6): 861-9. PMid:32142111. https://doi.org/10.1001/jamainternmed.2020.0664

[7] United Nations Office on Drugs and Crime, World Drug Report 2019

[8] Jonas AM, Raj R. Vaping-Related Acute Parenchymal Lung Injury: A Systematic Review. Chest. 2020; 158(4): 1555-65. PMid:32442559. https://doi.org/10.1016/j.chest.2020.03.085
[9] Butt YM, Smith ML, Tazelaar HD, et al. Pathology of VapingAssociated Lung Injury. N Engl J Med. 2019; 381(18): 1780-1. PMid:31577870. https://doi .org/10.1056/NEJMc1913069

[10] Marsden L, Michalicek ZD, Christensen ED. More on the Pathology of Vaping-Associated Lung Injury. N Engl J Med. 2020; 382(4): 3878. PMid:31747506. https://doi.org/10.1056/NEJMc1914980

[11] Mukhopadhyay S, Mehrad M, Dammert P, et al. Lung Biopsy Findings in Severe Pulmonary Illness Associated With E-Cigarette Use (Vaping). Am J Clin Pathol. 2020; 153(1): 30-9. PMid:31621873. https://doi.org/10.1093/ajcp/aqz182

[12] Shields PG, Song MA, Freudenheim JL, et al. Lipid laden macrophages and electronic cigarettes in healthy adults. EBioMedicine. 2020; 60: 102982. PMid:32919101. https://doi.org/10.1016/j.ebiom.2020.102982

[13] Blount BC, Karwowski MP, Shields PG, et al. Vitamin E Acetate in Bronchoalveolar-Lavage Fluid Associated with EVALI. N Engl J Med. 2020; 382(8): 697-705. PMid:31860793. https://doi.org/ 10.1056/NEJMoa1916433

[14] Kleinman MT, Arechavala RJ, Herman D, et al. E-cigarette or Vaping Product Use-Associated Lung Injury Produced in an Animal Model From Electronic Cigarette Vapor Exposure Without Tetrahydrocannabinol or Vitamin E Oil. J Am Heart Assoc. 2020; 9(18): e017368. PMid:32896206. https://doi.org/10.1161/JAHA.1 20.017368

[15] Colombo JL, Hallberg TK. Pulmonary aspiration and lipid-laden macrophages: in search of gold (standards). Pediatr Pulmonol. 1999; 28(2): 79-82. PMid:10423305. https://doi.org/10.1002/(SI CI) 1099-0496 (199908) 28:2<79: :AID-PPUL1>3.0.CO;2-A 